\title{
RELATIONSHIP BETWEEN WORKING MEMORY, FLUID INTELLIGENCE AND AGE BASED ON THE RESULTS OF MEDIATION AND MODERATION ANALYSES
}

\author{
Ludmiła Zając-Lamparska
}

Faculty of Psychology, Kazimierz Wielki University in Bydgoszcz

\begin{abstract}
The positive relationship between working memory (WM) and fluid intelligence (Gf) is a well-established phenomenon, yet numerous studies reveal the age-related decline in both WM and Gf. From the perspective of the changes that WM and Gf undergo in adult development it may be assumed that accounting for age in studies of the relationship between these two is important, particularly in age-diverse groups. However, the issue of the WM-Gf link has rarely been considered from a developmental perspective, especially in adults. The analyses presented here focused on the role of age in the relationship between WM and Gf. The study sample comprised 63 participants in early adulthood $(N=33$, aged 20-34) and late adulthood $(N=30$, aged 60-73). They performed a computerised n-back task on 6 difficulty levels (from 1-back to 6-back) and Raven's Standard Progressive Matrices
\end{abstract}

LudMILA ZaJąC-LAMPARSKA, https://orcid.org/0000-0003-4618-547X. Correspondence concerning this article can be addressed to Ludmila ZająC-LAmparsKa, Wydział Psychologii, Uniwersytet Kazimierza Wielkiego w Bydgoszczy, ul. Staffa 1, 85-867 Bydgoszcz, Poland; e-mail: lzajac@ukw. edu.pl.

Data for this paper were collected while qualifying subjects for a sample under the project "Compensatory brain activity in older adults. The search for the electrophysiological indicators of cognitive processes involved in this activity, and its possible changes induced by working memory training", funded by the National Science Centre, Poland (2017/25/B/HS6/00360). The study was not preregistered. The data supporting the findings here are available from the author upon reasonable request.

I would like to thank Hanna Bartosz, Monika Deja, and Łukasz Warchoł for participating in data collection.

Handling editors: Magdalena Szubielska \& Pawel StróżaK, John Paul II Catholic University of Lublin.

Received 7 April 2021. Received in revised form 24 June 2021, 24 Sept. 2021. Accepted 13 Oct. 2021. Published online 26 Nov. 2021. 
(SPM) in Polish standardisation. The obtained results indicated that although WM and Gf were found to be inferior among older than young adults, age was not a significant moderator of the association between WM and Gf. The relationship between age and Gf was not mediated by WM, which does not confirm that age-related changes in Gf may partially result from the developmental trajectory of WM. At the same time, age fully mediated the relationship between WM and Gf, suggesting the existence of a general factor of cognitive aging and implying that it may underlie an apparent relationship between WM and Gf in age-diverse groups.

Keywords: working memory; fluid intelligence; development; mediation; moderation.

\section{Relationship Between Working Memory and Fluid Intelligence}

The positive relationship between working memory (WM) and fluid intelligence (Gf) has been an issue explored in cognitive psychology for a long time, and its existence is now well-established. Intensive research on this problem was conducted at the turn of the 21 st century. There is a wide range of evidence of such a relationship from studies carried out in this period (e.g., Colom et al., 2003; Colom et al., 2006; Conway et al., 2002; Engle et al., 1999a; Engle et al., 1999b; Fukuda et al., 2010; Kane et al., 2007; Kyllonen \& Christal, 1990; Shelton et al., 2010; Süß et al., 2002). Moreover, the evidence is also supported by meta-analyses. Meta-analysis which included the results of 57 studies (86 samples) suggested an average correlation between WM and Gf of 0.48 (Ackerman et al., 2005). While a reanalysis of the same dataset yielded a much higher correlation estimate of $r=0.85$ (Oberauer et al., 2005). Similarly, a reanalysis focused on the outcomes of latent-variable studies found stronger correlation between WM capacity and Gf (median $r=0.72$ ), indicating that the WM capacity and Gf constructs share approximately $50 \%$ of their variance (Kane et al., 2005). A strong link between WM and Gf have also been confirmed by subsequent studies (e.g., Cochrane et al., 2019; Colom et al., 2015; Jastrzębski et al., 2018; Rey-Mermet et al., 2019; Rose, 2013; Smoleń $\&$ Chuderski, 2015; Thomas et al., 2015).

This link, observed at the behavioural level, is also supported by data on neuronal mechanisms shared by Gf and WM, primarily involving the dorsolateral prefrontal cortex, parietal cortex and fronto-parietal network (Barbey et al., 2014; Burgess et al., 2011; Dehn, 2017; Eriksson et al., 2015; Gray et al., 2003; Kane \& Engle, 2002).

Theoretical explanations of the nature of the relationship between WM and Gf have focused on the search for the lower-level mechanisms underlying the variance shared by these two constructs. Current theoretical understandings of this relationship are mostly based on two explanatory factors: (1) executive control also referred to as attention control or executive attention (Burgess et al., 2011; Cowan 
et al., 2006; Engle \& Kane, 2004; Engle, Tuholski et al., 1999; Nęcka \& Lulewicz, 2016; Shipstead et al., 2016) and (2) WM storage capacity (Chuderski et al., 2012; Cochrane et al., 2019; Colom et al., 2008; Conway et al., 2002; Rey-Mermet et al., 2019; Smolen \& Chuderski, 2015). In addition, as mechanisms underlying the WM-Gf relationship the updating of WM content (Chuderski \& Nęcka, 2012; Cochrane et al., 2019; Kane et al., 2007) and learning efficiency (Harrison et al., 2015; Wiley et al., 2011) are also mentioned. There is also evidence that the WM-GF relationship can be explained by several factors operating simultaneously, for example, WM storage capacity and executive/attention control (Chuderski \& Nęcka, 2012; Unsworth et al., 2009); short-term storage and executive functioning (Dang et al., 2014); WM storage capacity, executive/attention control and secondary memory abilities (Unsworth \& Spillers, 2010; Unsworth et al., 2014); the scope and control of attention (Shipstead et al., 2012). Kovacs and Conway (2016), in the context of their process overlap theory, conclude that the link between WM and Gf is driven by the operation of multiple domain-general cognitive processes which are involved in both groups of task, measuring Gf and WM. Despite years of research and numerous results confirming the relationship between WM and Gf, researchers have been unable to provide a conclusive answer as to why the two constructs are related, and the question of what explains this relationship remains open (Burgoyne et al., 2019; Frischkorn \& Oberauer, 2021; Harrison et al., 2015).

\section{The Role of Age in the Relationship Between Working Memory and Fluid Intelligence}

The issue of the relationship between WM and Gf has rarely been considered from a developmental perspective, especially in adults. It is noteworthy that nearly all studies proving the link between WM and Gf were conducted in the same age group, i.e., young adults. In fact, almost all studies cited above were conducted in this age group. The participants of the research on WM-Gf relationship were primarily undergraduate students, therefore, these samples are characterized by very low age variability (e.g., Burgoyne et al., 2019; Colom et al., 2005; Conway et al., 2002; Dang et al., 2014; Engle, Tuholski et al., 1999; Fukuda et al., 2010; Kane et al., 2007; Shelton et al., 2010; Rose, 2013; Wiley et al., 2011). Alternatively, the research involved persons aged between 18 and 30-35 (Chuderski, 2014; Chuderski \& Nęcka, 2012; Shipstead et al., 2012; Thomas et al., 2015; Unsworth et al., 2014; Unsworth \& Spillers, 2010). At the same time, the age variable was not taken into account in the conducted analyses. However, age was also not included in the ana- 
lyses when the age diversity of the participants was greater, e.g., 18 to 46 years of age (Chuderski, 2015; Süß et al., 2002) or 14 to 47 years of age (Colom et al., 2003).

The contemporary consensus in psychology is that both WM and Gf are affected by developmental changes, including developmental changes in adulthood. In adults, there is a decrease in WM and GF with age, which is particularly evident in late adulthood (Basak \& Verhaeghen, 2011; Braver \& West, 2008; Harada et al., 2013; Salthouse, 2009; Salthouse, 2016). According to Salthouse's (2011) findings based on the results from 3,000 adults, fluid ability deteriorates with age by an estimated -0.02 standard deviation per year between the ages of 20 and 70 . Similarly, a study involving a very large sample (nearly 50,000 participants) found that over the course of development, the peak in Gf-related ability is reached early, followed by a decline (Hartshorne \& Germine, 2015). A linear relationship between various working memory measures (each of them) and age were indicated by research conducted in adults in the age range 20-86 (Borella et al., 2008). Furthermore, a meta-analysis of 123 studies with the use of Brinley analysis revealed that working memory span is more age sensitive than short-term memory span (Bopp \& Verhaeghen, 2005). Similarly, a relatively recent meta-analysis, supplemented by Brinley and state-trace analyses, demonstrated a small differential effect of age on tasks requiring the temporary storage and processing of information (specific to WM), relative to storage alone (Jaroslawska \& Rhodes, 2019).

\section{Three Possible Roles of Age in the Relationship Between WM and Gf}

From the perspective of the changes that WM and Gf undergo in adult development it may be assumed that accounting for age in studies of the relationship between WM and Gf is important, particularly in age-diverse groups. The age variable in studies on the relationship between WM and Gf can be considered in (at least) three ways.

Firstly, age can be a moderator of the relationship between WM and Gf. The identification of the moderating role of age raises questions about the mechanism of age-related change not only in the individual components in the relationship but also in how they relate (Cochrane et al., 2019). However, in the previously mentioned meta-analysis by Ackerman, Beier, and Boyle (2005) there was no consistent indication of the moderating role of age for the WM-Gf relations based on studies involving participants aged between 13 and 70. Age also did not prove to be a significant moderator of the association between WM and Gf in the study involving children and young adults, leading to the conclusion that the hierarchical cognitive basis of intelligence is stable from childhood into adulthood (Cochrane et al., 2019) 
Secondly, age can be a predictor that influences Gf through WM, i.e., WM can mediate the relationship between age and Gf. It means that age-related changes in Gf may result from the developmental trajectory of WM. For example, a study of the developmental changes in Gf from childhood through adolescence to early adulthood revealed that almost half of the age-related increase of Gf was mediated by developmental changes in WM and processing speed (Fry \& Hale, 1996). In describing the obtained results, the authors use the concept of a developmental cascade, which captures well the nature of the mediated relationship in which age affects processing speed, which in turn affects WM, and finally WM affects Gf. Nettleback and Burns (2010) investigated whether such a developmental cascade (but reversed) occurs also in adulthood, including old age. Their study indicated that results obtained among adults resembled a cascade with diminishing reasoning ability mediated by processing speed and working memory. However, it was not the literal inverse of the cascade observed at a young age. The mediating role of WM in the relationship between age and reasoning ability was confirmed, whereas the main differences with adults were a direct causal path between age and working memory and a lesser role of processing speed. According to the author's conclusions, declining reasoning ability in old age is influenced by a WM decrease that only partially results from slower processing speed. The mediating role of WM in the effect of age on Gf was also indicated by other studies (Manard et al., 2014; Salthouse, 1991; Schretlen et al., 2000). At the same time, the mediating role of WM in the relationship between age and Gf is not unambiguous and well documented. In fact, there are not many studies that are strictly devoted to this issue.

Finally, in age-mixed groups, there may be increased correlations between WM and Gf as a function of the concomitant changes in both WM and Gf with age. In such cases, age can operate as a confounding factor, the so-called lurking variable. This means that age can be a source of spurious relationship or apparent correlation between WM and Gf, or at least a cause for overestimating the strength of this relationship. Results of an extensive meta-analysis indicate that a significant range of changes occurring with age are shared by a variety of cognitive abilities, with the proportion of these shared changes increasing from about $45 \%$ at age 35 to about $70 \%$ at age 85 (Tucker-Drob et al., 2019). This suggests that in groups that are age-diverse and include older adults, the risk of overestimating a correlation or the occurrence of an apparent correlation increases. In some studies on the relationship between WM and GF, the influences of age were statistically controlled by including age as a predictor in the analyses and partialling the effects associated with age from each variable (Fry \& Hale, 1996; Salthouse, 2014; Salthouse \& Pink, 2008). However, many studies conducted in age-diverse groups, including the studies mentioned in this article, failed to control for the contribution of age in the WM-Gf relationship. 
The purpose of the analyses presented in this paper was to test all three possible positions of age in the relationship between WM and Gf: (1) age as a moderator of WM-Gf relationship; (2) age as an independent variable that affects Gf indirectly, mediated by WM; (3) age as a lurking variable, forming the spurious relationship between WM and Gf.

\section{METHOD}

\section{Participants}

The sample comprised 63 volunteers, including 33 young adults (aged 20-34, $M=24.91, S D=4.10 ; 22$ women) and 30 older adults (aged 60-73, $M=66.17$, $S D=3.55 ; 25$ women). The exclusion criteria were as follows: mental illness, history of neurological disorders, including neurodegenerative diseases or severe head injuries, intellectual disability, dementia symptoms (in older adults), and uncorrected vision defects. The study sample demonstrated a large disproportion in terms of gender, but the analyses conducted proved that gender does not differentiate the variables examined in both age groups. ${ }^{1}$

\section{Instruments}

As a measure of Gf, Raven's Standard Progressive Matrices (SPM) test in Polish standardisation was employed (Jaworowska \& Szustrowa, 2000). There were no time limits for completing the SPM test. ${ }^{2}$ Raw SPM scores were used as an indicator of Gf.

${ }^{1}$ Mann-Whitney $U$ test for Gf measure in older adults group: $U=58.00 ; z=0.22 ; p=.824$ and in young adults group: $U=76.00 ; z=-1.70 ; p=.089$; Mann-Whitney $U$ test for the indicator of WM task performance in older adults group: $U=48.00 ; z=-0.78 ; p=.436$ and in young adults group: $U=120.00 ; z=0.019 ; p=.985$

${ }^{2}$ Although there were no time limits for completing the SPM test, the time taken to complete it was measured. It was longer $(t=2.90 ; p=.005)$ in older adults $(M=38.28 \mathrm{~min} ., S D=13.76)$ than in young adults $(M=29.47 \mathrm{~min}$., $S D=8.54)$. At the same time, there was no relationship between the SPM test completing time and working memory performance in older adults $(r=-0.13 ; p=.534)$ and young adults $(r=0.03 ; p=.860)$. It can therefore be derived that a longer time spent completing the SPM test does not impair performance on the task measuring WM. In contrast, there was a significant positive correlation between the SPM test completing time and performance on this test in older adults $(r=0.53 ; p=.006)$ and young adults $(r=0.47 ; p=.009)$. 
For the assessment of WM, a single n-back task at 6 levels of difficulty (from 1-back to 6-back) was used. The n-back task is a commonly used measure of working memory efficiency in terms of its content updating (Jaeggi et al., 2010; Schmiedek et al., 2014). The n-back task consists of continuous presentation of items (letter in the present research) that appear and disappear one by one. During each presentation, the participant has to judge whether the currently displayed item matches the item presented $n$ trials back. Thus, the letter ' $n$ ' in the n-back task indicates to which item (presented how many trials earlier) the currently presented item should be compared. In the research presented here, the currently presented item had to be compared to an item presented 1, 2, 3, 4, 5 or 6 trials back, depending on the difficulty level. The rules for the n-back task are illustrated in Figure 1, using the example of the 2-back task.

\section{Figure 1}

Single N-Back Task Paradigm (Example: 2-Back)
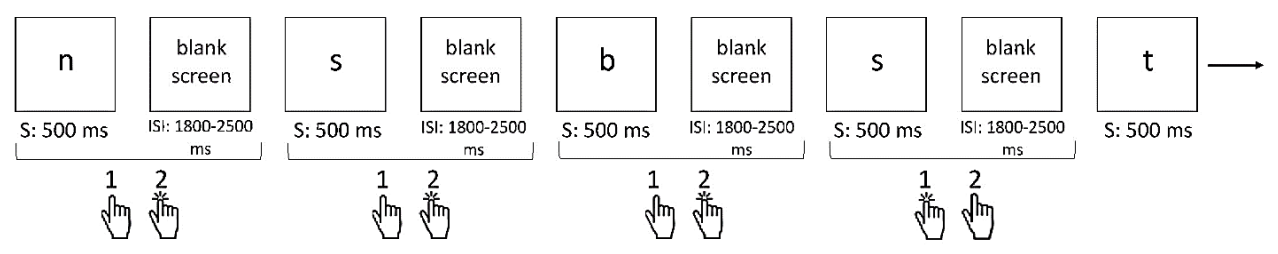

Note. Description of 2-back task: On the computer screen the letters appear and disappear one by one. The task is to decide whether the currently presented letterhas appeared 2 items back. Ifyes, the respondent should press the "1" button on the response pad; if no, the "2" button. For the sequence of letters shown in the illustration: $\mathrm{n}, \mathrm{s}, \mathrm{b}, \mathrm{s}, \mathrm{t}$, the following sequence of reactions (button selections) is correct: 2, 2, 2, 1, 2. $\mathrm{S}=$ stimulus presentation, ISI = inter-stimulus interval.

In the context of the relationship between WM and Gf, the n-back task is one of two relatively simple WM tasks, which successfully predicted intellectual abilities. The second one is running memory task (Chuderski \& Nęcka, 2012). N-back task measures two aspects of WM: capacity and executive control (Nęcka \& Lulewicz, 2016). At the cognitive level, this task requires not only active maintenance of items in WM, but also the updating of new items so that they can be actively maintained, tracking the serial order and the rapid binding of items to their serial order so that responses are based on the match between the current item and the n-back item, and resolution of the non-n-back items interference (Burgess et al., 2011; Chatham et al., 2011). Research using event-related potentials supported at the neurophysiological level the three-sub-process model of the n-back task (Chen et al., 2008). This model 
indicates matching, replacement and shift as sub-processes involved in the n-back task, starting from 2-back level (for 1-back only matching and replacement are necessary). According to the obtained results, replacement is a data-driven process with a posterior locus whereas shift is a more conceptual process with a more frontal locus. Moreover, neuroimaging data indicated that activity in lateral prefrontal and parietal regions, discriminating for high Gf scores, was obtained also in an n-back task (Gray et al., 2003). Finally, a latent factor analysis of WM measures using large-scale data revealed that n-back tasks had the highest loadings on the general WM factor (Waris et al., 2017). According to our conclusions, this general WM factor can reflect high-level cognitive resources, such as executive functioning and fluid intelligence, that are involved in all WM tasks, and possibly even more in the n-back task.

The n-back task used in the presented research was programmed in PsychoPy software (Peirce, 2009). Each difficulty level included 4 blocks. In each block, $20+n$ (where $n$ denotes the number from the term ' $n$-back') stimuli were presented, including 7 targets, i.e., the same items as those presented $\mathrm{n}$ trials back. The stimulus presentation duration was $500 \mathrm{~ms}$, while the inter-stimulus interval (ISI) was randomised from 1800 to $2500 \mathrm{~ms}$. Before the actual task at each level of difficulty, the participant had a short training session to learn the operating rules. The indicators of the n-back task performance at each level of difficulty were: rate of correct responses to targets, rate of false alarm errors and sensitivity index ( $\left.\mathrm{d}^{\prime}\right)$, as a measure of discriminability based on signal detection theory. Sensitivity index ( $\left.\mathrm{d}^{\prime}\right)$ was calculated according to the formula for yes/no tasks: $d^{\prime}=z(\mathrm{H})-z(\mathrm{FA})$, where $\mathrm{H}$ and FA are the Hits and False Alarm rates, respectively, and $z(\mathrm{H})$ and $z(\mathrm{FA})$ are the $z$-transformations (Stanislaw \& Todorov, 1999).

In addition, the following instruments were used to assess the absence of exclusion criteria: a structured interview constructed for the purpose of the study, and a structured diagnostic interview: a Polish version of the Mini International Neuropsychiatric Interview - the M.I.N.I. Screen 7.0.2 (Sheehan et al., 1998), and in older adults only: the Mini Mental State Examination-MMSE (Stańczak, 2010) as well as the Addenbrooke's Cognitive Examination III-ACE III (Hodges et al., 2017).

\section{Procedure}

The study protocol and the informed consent form were approved by the Bioethics Committee of the Nicolaus Copernicus University in Torun at the Ludwik Rydygier Collegium Medicum in Bydgoszcz (KB 90/2018). Before enrolment in 
the study, each participant was informed about its purpose, anonymity, and the possibility of withdrawal from the study. All subjects gave written informed consent in accordance with the Declaration of Helsinki. The research was performed with each participant individually. The order in which the tools were used was fixed. The assessment began with a structured interview constructed for the purpose of the study, and then the M.I.N.I. screen was carried out, followed by MMSE and ACE III administration, but only in older adults. The next tool used was the SPM. And finally, the participants performed the n-back task, sequentially at 6 levels of difficulty.

\section{Data Analyses}

The comparison between the group of young and older adults in terms of Gf (SPM raw scores) was made using Student's $t$ test. A comparison of age groups in regard to working memory (rate of correct responses to targets, rate of false alarm errors and $\mathrm{d}^{\prime}$ for the n-back task at 6 levels of difficulty) was performed using Hotelling's T-Squared test, supplemented by Student's $t$ tests. The association between raw scores on the SPM test and performance on the n-back task at each level of difficulty was assessed using Pearson's $r$ correlation coefficients. The comparison of the n-back task performance in age groups as well as the correlation analyses were supplemented with the False Discovery Rate (FDR) correction for multiple comparisons. To investigate whether age moderates the relationship between WM and Gf, a hierarchical multiple regression analysis was conducted. To test whether WM mediates the effect of age on Gf, and to test whether age can function as a source of apparent correlation between WM and Gf, two separate mediation analyses were conducted. In the comparison of age groups and in the moderation analysis, age was treated as a dichotomous nominal variable (young adults vs. older adults). In correlation analyses and mediation analyses, age was a quantitative, continuous variable (expressed in years). All n-back task performance indicators in all analyses are quantitative, continuous variables. Moreover, for all the performed statistical analyses, the sensitivity power analyses were conducted to determine the required population effect that can be reliably detected in the context of effect sizes reported in the literature. Descriptive statistics, correlation analyses, Student's t-tests and Hotelling's T-squared test were made using Statistica 13.1 PL software. Moderation and mediation analyses were carried out using PROCESS macro with model 1 for mediation analyses and model 4 for moderation analysis (Hayes, 2018). The sensitivity power analyses were conducted using G*Power 3.1.9.4. (Faul et al., 2007). Excel was used to calculate d' and FDR correction. 


\section{RESULTS}

\section{Age Groups Comparison in Terms of Fluid Intelligence and Working Memory}

The obtained results indicated that older adults achieved lower raw score in SPM than young adults. They also revealed worse n-back performance when the sensitivity index $\left(\mathrm{d}^{\prime}\right)$ was used for comparison. The differences were statistically significant for the task difficulty levels: 1-, 2- and 5-back, and reached the level of statistical tendency for the rest of difficulty levels. Further analysis, including the FDR correction, showed that inferior $\mathrm{d}^{\prime}$ indicator in older adults did not result from lower correctness of the response to the target, but a higher rate of false alarms in this group - except from the easiest level of the n-back task (1-back). The results of the analyses concerning the comparison of age groups in terms of WM and Gf are presented in Table 1.

Determining the strength of an effect that can be reliably detected, conducted with the use of the sensitivity power analysis, revealed that with $\alpha$ of 0.05 , desired statistical power of 0.80 , and with present sizes of the age groups, the minimum detectable effect size (Cohen's $d$ ) was 0.634 , which means medium effect size (Lakens, 2013). Based on the literature, we should expect at least medium effects (Jaroslawska \& Rhodes, 2019; Salthouse, 2009; (Tucker-Drob et al., 2019), but including those below the lower detectable effect size. Thus, the present study could be underpowered for purposes of detecting some differences between age groups in terms of correct answers to the targets for which the effect was medium but lower than 0.634. At the same time, the effect sizes obtained for age-related differences in the rate of correct answers to the targets are lower than for the differences in $\mathrm{d}^{\prime}$, and substantially lower than for the differences in the rate of false alarms. This supports the finding of a difference between older and young adults, manifested in $\mathrm{d}^{\prime}$, which is mainly derived from false alarms. 


\section{Table 1}

Comparison of Age Groups on Fluid Intelligence and Working Memory

\begin{tabular}{|c|c|c|c|c|c|c|c|c|}
\hline & $\begin{array}{c}\text { Young adults } \\
M(S D)\end{array}$ & $\begin{array}{c}\text { Older adults } \\
M(S D)\end{array}$ & $\begin{array}{c}\text { Student's } \\
T\end{array}$ & $p$ & $q$ & $\begin{array}{c}\text { Cohen's } \\
d\end{array}$ & $\begin{array}{c}\text { Hotelling } \\
T^{2}\end{array}$ & $p$ \\
\hline SPM & $53.39(4.37)$ & $42.13(9.57)$ & 6.10 & $<.001$ & & 1.51 & & \\
\hline \multicolumn{9}{|c|}{ n-back: d' } \\
\hline 1-back & $3.28(0.83)$ & $2.58(0.82)$ & 3.35 & .001 & .004 & 0.85 & 23.04 & .005 \\
\hline 2-back & $2.43(0.84)$ & $1.76(0.64)$ & 3.57 & $<.001$ & .002 & 0.90 & & \\
\hline 3-back & $1.55(0.57)$ & $1.27(0.44)$ & 2.13 & .037 & .066 & 0.55 & & \\
\hline 4-back & $1.28(0.49)$ & $1.03(0.51)$ & 2.02 & .047 & .066 & 0.50 & & \\
\hline 5-back & $1.07(0.49)$ & $0.76(0.44)$ & 2.60 & .011 & .027 & 0.67 & & \\
\hline 6-back & $1.02(0.50)$ & $0.73(0.61)$ & 2.06 & .043 & .066 & $0.5 \mathrm{e} 2$ & & \\
\hline \multicolumn{9}{|c|}{ n-back: correct responses to targets (rate) } \\
\hline 1-back & $0.92(0.10)$ & $0.86(0.11)$ & 2.34 & .023 & .045 & 0.57 & 10.47 & 0.16 \\
\hline 2-back & $0.80(0.15)$ & $0.79(0.14)$ & 0.25 & .802 & .802 & 0.07 & & \\
\hline 3-back & $0.66(0.16)$ & $0.71(0.16)$ & -1.39 & .170 & .191 & 0.31 & & \\
\hline 4-back & $0.57(0.16)$ & $0.65(0.20)$ & -1.83 & .073 & .087 & 0.44 & & \\
\hline 5-back & $0.51(0.15)$ & $0.56(0.19)$ & -1.17 & .244 & .258 & 0.29 & & \\
\hline 6-back & $0.46(0.16)$ & $0.55(0.21)$ & -2.02 & .048 & .066 & 0.48 & & \\
\hline \multicolumn{9}{|c|}{ n-back: false alarms (rate) } \\
\hline 1-back & $0.06(0.08)$ & $0.11(0.11)$ & -1.95 & .055 & .071 & 0.52 & 56.08 & $<.001$ \\
\hline 2-back & $0.09(0.06)$ & $0.22(0.11)$ & -6.21 & $<.001$ & $<.001$ & 1.47 & & \\
\hline 3-back & $0.15(0.07)$ & $0.27(0.11)$ & -5.29 & $<.001$ & $<.001$ & 1.30 & & \\
\hline 4-back & $0.15(0.07)$ & $0.30(0.13)$ & -5.78 & $<.001$ & $<.001$ & 1.44 & & \\
\hline 5-back & $0.16(0.09)$ & $0.30(0.13)$ & -4.73 & $<.001$ & $<.001$ & 1.25 & & \\
\hline 6-back & $0.15(0.10)$ & $0.30(0.13)$ & -5.12 & $<.001$ & $<.001$ & 1.29 & & \\
\hline
\end{tabular}

Note. SPM $=$ Raven's Standard Progressive Matrices test (raw scores); $q$ = adjusted values for $p$ using False Discovery Rate (FDR) correction for multiple comparisons.

\section{Relationship Between Working Memory and Fluid Intelligence in the Whole Sample and in Age Groups}

The revealed correlations between $\mathrm{d}^{\prime}$ in the n-back task and SPM raw scores were significant only when we analyse them for both age groups together (Table 2). When the two groups were analysed separately and when using the FDR correction, 
correlations ceased to be significant. The magnitude of correlation coefficients also decreased when they were evaluated separately in the age groups. A rather similar pattern could be seen for the relationship between SPM raw scores and false alarm rates. For the whole sample, all correlation coefficients were statistically significant, but this was no longer the case for age groups considered separately and using the FDR correction. In terms of correct responses to targets, these did not correlate significantly with SPM raw scores, either in the whole sample or in the age groups. The only exception was the correctness of responses to the target at 1-back level, which was significantly associated with the SPM performance when both age groups were considered together.

\section{Table 2}

Correlations Between Sensitivity Index for N-Back Task Performance and SPM Raw Scores

\begin{tabular}{|c|c|c|c|c|c|c|c|c|c|}
\hline & \multicolumn{3}{|c|}{ Whole sample } & \multicolumn{3}{|c|}{ Young adults } & \multicolumn{3}{|c|}{ Older adults } \\
\hline & Pearson's $r$ & $p$ & $q$ & Pearson's $r$ & $p$ & $q$ & Pearson's $r$ & $p$ & $q$ \\
\hline \multicolumn{10}{|c|}{$\mathrm{d}^{\prime}-\mathrm{SPM}$} \\
\hline 1-back & 0.39 & .001 & .004 & 0.17 & .357 & .536 & 0.25 & .180 & .360 \\
\hline 2-back & 0.41 & .001 & .002 & 0.19 & .280 & .536 & 0.27 & .156 & .360 \\
\hline 3-back & 0.24 & .055 & .082 & 0.11 & .536 & .634 & 0.13 & .507 & .812 \\
\hline 4-back & 0.23 & .075 & .104 & 0.47 & .006 & .112 & -0.08 & 679 & .815 \\
\hline 5-back & 0.30 & .019 & .031 & 0.38 & .029 & .175 & 0.02 & .928 & .928 \\
\hline 6-back & 0.39 & .002 & .004 & 0.33 & .058 & .175 & 0.31 & .097 & .360 \\
\hline \multicolumn{10}{|c|}{ Correct responses to targets (rate) - $\mathrm{SPM}$} \\
\hline 1-back & 0.35 & .005 & .011 & 0.13 & .448 & .587 & 0.28 & .138 & .360 \\
\hline 2-back & 0.12 & .365 & .438 & 0.18 & .301 & .536 & 0.10 & .587 & .812 \\
\hline 3-back & -0.10 & .453 & .509 & -0.02 & .895 & .948 & 0.04 & .850 & .900 \\
\hline 4-back & -0.12 & .360 & .438 & 0.27 & .113 & .343 & -0.06 & .742 & .835 \\
\hline 5-back & -0.08 & .541 & .573 & 0.34 & .056 & .175 & -0.11 & .557 & .812 \\
\hline 6-back & -0.03 & .816 & .816 & 0.40 & .021 & .175 & 0.08 & .680 & .815 \\
\hline \multicolumn{10}{|c|}{ False alarms (rate) - SPM } \\
\hline 1-back & -0.34 & .006 & .011 & -0.10 & .563 & .634 & -0.31 & .093 & .360 \\
\hline 2-back & -0.60 & $<.001$ & $<.001$ & -0.17 & .345 & .536 & -0.41 & .023 & .311 \\
\hline 3-back & -0.53 & $<.001$ & $<.001$ & -0.25 & .156 & .351 & -0.30 & .114 & .360 \\
\hline 4-back & -0.47 & $<.001$ & $<.001$ & -0.35 & .047 & .175 & -0.11 & .565 & .812 \\
\hline 5-back & -0.47 & $<.001$ & $<.001$ & -0.13 & .456 & .587 & -0.26 & .171 & .360 \\
\hline 6-back & -0.52 & $<.001$ & $<.001$ & -0.01 & .958 & .958 & -0.39 & .035 & .311 \\
\hline
\end{tabular}

Note. SPM $=$ Raven's Standard Progressive Matrices test (raw score) $q=$ adjusted values for $p$ using False Discovery Rate (FDR) correction for multiple comparisons. 
The sensitivity power analysis found that with $\alpha$ of 0.05 , a desired statistical power of 0.80 , and with the present sample size, the lower critical $r$ for the whole sample was 0.21 ; for the older adults' group it was 0.31 , and for young adults group it was 0.29 . This means that with the present sample size, weak correlations were not detectable (Akoglu, 2018). At the same time, the critical detectable correlation coefficients are clearly lower than the correlations of 0.72 and 0.85 reported in the previously mentioned meta-analyses on the relationship between Gf and WM (Kane et al., 2005; Oberauer et al., 2005).

\section{Age as a Moderator of the Relationship Between Working Memory and Fluid Intelligence}

In the first step of a hierarchical multiple regression analysis, two variables were included: WM (mean d' value for n-back task at 6 levels of difficulty) and age (as a dichotomous nominal variable, young adults vs older adults). These variables accounted for a significant amount of variance in Gf, $R^{2}=0.438, f^{2}=0.779$, $F(2,60)=22.377, p<.001$. To avoid potentially problematic high multicollinearity with the interaction term, the variables were mean-centred and an interaction term between WM and age was created (Aiken \& West, 1991; Hayes, 2018). Next, the interaction term between WM and age was added to the regression model (Table 3 ).

Table 3

Model Including Age Variable as Moderator of Relationship Between WM and Gf

\begin{tabular}{lcccc}
\hline $\begin{array}{l}\text { Model summary: } \\
R^{2}=0.440, f^{2}=0.786, \\
M S E=49.963,\end{array}$ & Coeff. & SE & $t$ & $p$ \\
$F(3,59)=12.683, p<.001$ & & & & \\
\hline Constant & 48.249 & 1.035 & 46.638 & $<.001$ \\
Age group $(M)$ & -8.586 & 2.074 & -4.140 & $<.001$ \\
n-back d' $(X)$ & 6.335 & 2.535 & 2.499 & .015 \\
$X \times M$ & 2.093 & 5.079 & 0.412 & .682 \\
\hline
\end{tabular}

Note. $\mathrm{n}$-back d' $=$ mean $\mathrm{d}^{\prime}$ value for $\mathrm{n}$-back task at 6 levels of difficulty.

The interaction age $\mathrm{x}$ WM accounted for an insignificant proportion of the variance in Gf: $\Delta R^{2}=0.002, \Delta f^{2}=0.006, \Delta F(1,59)=0.170, p=.682$. Examination of the interaction plot showed a rather comparable positive association between WM and Gf in both age groups (Figure 2). The obtained results did not confirm that age serves as a moderator of the association between WM and Gf. According to the 
sensitivity power analysis, with $\alpha$ of 0.05 , a desired statistical power of 0.80 , and with present sample size, the lower detectable $f^{2}$ for model including moderator was 0.185 , which is lower than the $f^{2}$ of the obtained model (Table 3 ).

Figure 2

Association Between Working Memory and Fluid Intelligence in Early and Late Adulthood Age Groups

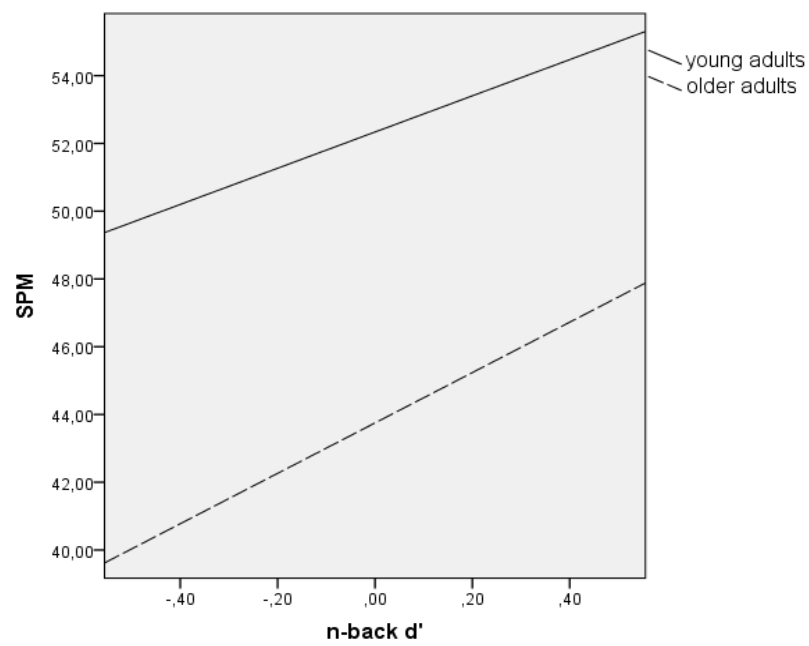

Note. SPM = Raven's Standard Progressive Matrices test (raw score); n-back d'= mean d' value for n-back task at 6 levels of difficulty.

\section{Working Memory as a Mediator of the Relationship Between Age and Fluid Intelligence}

In the first mediation analysis, age (as a continuous variable, expressed in years) was treated as the independent variable, fluid intelligence (indicated by the raw score of the SPM) as the dependent variable, and working memory (indicated by the mean $\mathrm{d}^{\prime}$ for all levels of the n-back task performance) as the mediator. The variables were mean-centred. The results of this model are shown in Table 4. 


\section{Table 4}

Model Coefficients for Model of Relation of Age to Fluid Intelligence With Working Memory as Mediator

\begin{tabular}{|c|c|c|c|c|c|c|c|c|c|c|}
\hline \multirow[b]{3}{*}{ Antecedent } & \multicolumn{10}{|c|}{ Consequent } \\
\hline & \multicolumn{5}{|c|}{$M\left(n-b a c k d^{\prime}\right)$} & & \multicolumn{4}{|c|}{ Y (fluid intelligence) } \\
\hline & & Coeff. & $S E$ & $t$ & $p$ & & Coeff. & $S E$ & $t$ & $p$ \\
\hline $\mathrm{X}$ (age) & $a$ & -0.010 & 0.002 & -4.698 & $<.001$ & $c^{\prime}$ & -0.231 & 0.056 & -4.104 & $<.001$ \\
\hline$M\left(n-b a c k d^{\prime}\right)$ & & & & & & $b$ & 5.377 & 3.450 & 1.558 & .124 \\
\hline \multirow[t]{3}{*}{ Constant } & $i_{M}$ & 2.036 & 0.110 & 18.530 & $<.001$ & $i_{Y}$ & 47.467 & 5.631 & 8.429 & $<.001$ \\
\hline & \multicolumn{5}{|c|}{$R^{2}=0.285 ; f^{2}=0.403$} & \multicolumn{5}{|c|}{$R^{2}=0.473 ; f^{2}=0.898$} \\
\hline & \multicolumn{5}{|c|}{$F(1,61)=22.073, p<.001$} & \multicolumn{5}{|c|}{$F(2,60)=18.260, p<.001$} \\
\hline
\end{tabular}

Note. $\mathrm{X}=$ independent variable, $\mathrm{Y}=$ dependent variable, $\mathrm{M}=$ mediator.

In the first step the significance of the relationship between age and fluid intelligence was confirmed. The regression of the age on the fluid intelligence, ignoring the mediator, was significant: $b=-0.287, t(61)=-6.812, p<.001, R^{2}=0.432$, $f^{2}=0.761$. Step two showed that the regression of the age on the mediator, working memory, was also significant. The third step of the mediation process showed that the mediator, working memory, controlling for age was not significant. The fourth step of the analyses, in turn, revealed that controlling for the mediator, age was still a significant predictor of fluid intelligence (see Table 4). As regards an indirect effect of age on fluid intelligence, the effect size was 0.056 . A bootstrap $95 \%$ confidence interval based on 5,000 bootstrap samples included zero $(-0.140,0.007)$, meaning that there was no evidence of an indirect effect of age on fluid intelligence through working memory. In addition, a Goodman test was also insignificant: $(Z=-1.516$, $p=.130)$. Taken together, the results indicated that working memory does not mediate the relationship between age and fluid intelligence. The sensitivity power analysis revealed that with $\alpha$ of 0.05 , a desired statistical power of 0.80 , and present sample size, the lower detectable effect size for regression of the independent on the dependent variable, ignoring the mediator was $f^{2}=0.100$, the same as for regression of the mediator on the dependent variable, whereas for regression including the independent variable and the mediator it was $f^{2}=0.161$. Effect sizes obtained for both models in the research are higher than the critical detectable effect sizes. 


\section{Age as a Variable Forming the Spurious Relationship Between Working Memory and Fluid Intelligence}

The second mediation model was intended to test whether age could operate as a "lurking" variable - a source of spurious relationship between working memory and fluid intelligence. For this purpose, this model treated age (as a continuous variable, expressed in years) as the mediator in the link between working memory (mean d' of n-back tasks) and fluid intelligence (SPM raw score). The variables were mean-centred. The results of this model are presented in Table 5.

Table 5

Model Coefficients for Model of Relation of Working Memory to Fluid Intelligence With Age as Mediator

\begin{tabular}{|c|c|c|c|c|c|c|c|c|c|c|}
\hline \multirow[b]{3}{*}{ Antecedent } & \multicolumn{10}{|c|}{ Consequent } \\
\hline & & \multicolumn{4}{|c|}{ M (age) } & & \multicolumn{4}{|c|}{ Y (fluid intelligence) } \\
\hline & & Coeff. & $S E$ & $t$ & $p$ & & Coeff. & $S E$ & $t$ & $p$ \\
\hline$X($ n-back d') & $a$ & -27.404 & 4.567 & -6.001 & $<.001$ & $c^{\prime}$ & 5.377 & 3.450 & 1.558 & .124 \\
\hline M (age) & & & & & & $b$ & -0.231 & 0.056 & -4.104 & $<.001$ \\
\hline \multirow[t]{3}{*}{ Constant } & $i_{M}$ & 87.673 & 7.848 & 11.171 & $<.001$ & $i_{Y}$ & 49.856 & 7.228 & 6.897 & $<.001$ \\
\hline & \multicolumn{5}{|c|}{$R^{2}=0.285 ; f^{2}=0.403$} & \multicolumn{5}{|c|}{$R^{2}=0.473 ; f^{2}=0.898$} \\
\hline & \multicolumn{5}{|c|}{$F(1,61)=36.011, p<.001$} & \multicolumn{5}{|c|}{$F(2,60)=18.260, p<.001$} \\
\hline
\end{tabular}

Note: $\mathrm{X}=$ independent variable, $\mathrm{Y}=$ dependent variable, $\mathrm{M}=$ mediator.

The regression analysis results confirmed the significance of the relationship between working memory and fluid intelligence, ignoring mediator: $b=11.701$, $t(61)=4.786, p<.001, R^{2}=0.273, f^{2}=0.376$. The regression of working memory on age as the mediator was also significant. Going further, the age mediator, controlling for working memory, was a significant predictor of fluid intelligence. In contrast, working memory, controlling for the mediator, was not (see Table 5). The effect size for an indirect effect of age on fluid intelligence was 6.325 and a bootstrap 95\% confidence interval based on 5,000 bootstrap samples did not include zero $(3.221 ; 10.983)$, which proved the contribution of age to the relationship between working memory and fluid intelligence. Moreover, a Goodman test was significant: $(Z=-3.432, p<.001)$. To summarise, these results revealed full mediation, indicating that the relationship between WM and Gf results from the association of both with age. Results of the sensitivity power analysis were the same as for the previous mediation analysis, 


\section{DISCUSSION}

\section{Comparison Between Older and Young Adults in Terms of Gf and WM}

In line with our expectations and the results of numerous previous studies, older adults revealed poorer performance on tasks measuring Gf and WM in comparison to young adults. With regard to WM, the source of poorer performance in older participants was mainly the false alarm errors. The analysis leading to this conclusion was underpowered, which may have led to a failure to detect the significance of differences between young and older adults, primarily in terms of correct responses to the target. However, even if these differences (or part of them) were to prove significant, the effect sizes obtained clearly indicate that the poorer $\mathrm{d}^{\prime}$ index of n-back task performance in older adults was mainly the result of the more frequent false alarm errors in this group.

This result can be interpreted in terms of the inhibitory deficit theory (Hasher \& Zacks, 1988). This theory explains the deterioration of cognitive functioning in old age by a decrease in inhibitory control of WM content. Ineffective inhibition may manifest itself in the situation of allowing inadequate information into WM, in retaining in WM information that is no longer meaningful, and the inability to inhibit imposing but inadequate responses (Lustig et al., 2007). The higher rate of false alarm errors in older adults is also consistent with the prefrontal-executive theory of ageing (West, 1996). According to this theory, the integration function of the prefrontal cortex is supported by four secondary processes, one of which is inhibition of an imposing reaction. As the prefrontal cortex is an area especially sensitive to morphological and functional changes accompanying ageing, cognitive ageing will be manifest in deficits of the aforementioned secondary processes, including inhibition (Cabeza \& Dennis, 2012). Finally, false alarm errors and inhibition deficits can be explained by the phenomenon of goal neglect, as assumed by goal maintenance theory (Braver \& West, 2008). However, the hits and false alarms rates can also reflect the general tendency to respond "yes" or "no". From this perspective, older adults would be more likely than young adults to answer "yes". In the context of signal detection theory, this can be understood as avoiding omission errors, even at the cost of false alarms. This tendency may be driven by some metacognitive strategy used by older adults, e.g., given the belief that memory declines with age and so does own experience with memory deterioration, older adults may choose to report the recognition of a signal despite a lower degree of confidence in the correctness of the recognition. In line with this point of view are results of research on the relationship between memory and post-decision confidence ratings among 
young and older adults. They revealed similar metacognitive efficiency (indicated by meta-d $\left.\mathrm{d}^{\prime} / \mathrm{d}^{\prime 3}\right)$ despite a significantly lower memory performance in older adults (Zakrzewski et al., 2021). It supports the idea that older adults can decide to identify signal presence, being aware of the uncertainty of this response. Also, in the study that took as its starting point the conceptual similarity between metacognition and executive function (EF), it turned out that EF steeply declined with age, while metacognitive efficiency was preserved. Moreover, according to the exploratory factor analysis results, EF and metacognition are loaded by different factors across all ages (Filippi et al., 2020).

On the other hand, research on effects of ageing on monitoring of retrieval revealed that older adults are sometimes more likely to produce high-confidence false alarms, which has been interpreted as a result of a poorer quality of memories causing high-confidence misrecollections (Dodson et al., 2007; Hertzog \& Dunlosky, 2011). This interpretation is in line with the misrecollection account, which suggests that misrecollections in older adults are partly caused by disinhibited binding processes (implicating miscombine features from separate events that occur in close temporal proximity) and by retrieval cues that activate features from non-desired but similar memories (Dodson et al., 2007). The misrecollection account obviously refers to more complex memories than a sequence of letters; however, the mechanisms it identifies can also occur during the n-back task performance.

In the absence of clear indications as to the interpretation of the obtained results, the possibility of a link between false alarm errors and the particular metacognitive strategy used by older adults requires further research.

\section{Lack of Moderating Role of Age in the Relationship Between WM and Gf}

Although age clearly differentiated the scores obtained in tasks measuring WM and Gf, it did not differentiate the relationship between them. It means that age was not found to be a significant moderator in the relationship between WM and Gf. This result suggests that the presence of the WM-Gf link is a constant phenomenon in adult development and that the nature of this relationship does not change with age during adulthood.

\footnotetext{
${ }^{3}$ Metacognitive efficiency is calculated by dividing meta-d' (i.e., the degree to which a participant's confidence ratings can discriminate between correct and incorrect responses) by d' (i.e., sensitivity index calculated on the basis of task performance). The division result equal to 1 indicates that metacognitive sensitivity (meta-d') matches task sensitivity (d') (Zakrzewski et al., 2021).
} 


\section{Two Alternative Mediation Analyses: Age as an Independent Predictor of Gf and Source of the Relationship Between WM and Gf}

Only one of the two models tested revealed significant mediation effect. The relationship between age and Gf mediated by WM, indicated in earlier studies, did not occur in the analysis presented in the article. In fact, a Gf variance appeared to be directly related to age, without the contribution of WM. Including $\mathrm{WM}$ as a mediator in the model increased the range of explained variance of Gf to a not very large extent (4\%). In contrast, full mediation was revealed in a model that examined the potential role of age as a lurking variable in the relationship between WM and Gf. In this case, when age as the mediator was included in the model, the increase in explained variance of Gf was clearly larger, that is, $18 \%$. This indicates that part of the association between WM and Gf may actually be derived from the relation of both these variables to age. In line with this interpretation are the results of the correlation analyses, which were found to be significant when conducted in the whole sample, and non-significant or rarely significant when conducted in age groups separately.

When considering the results of the two mediation analyses presented in this paper, it is important to note that the two models tested are not completely distinct, as in fact they differ only in the role assigned to two variables: age and WM. Therefore, the same group of relationships is analysed in both models, albeit in a different configuration. However, this distinction of configurations is crucial because they differ in the assumed relationships between the three variables: Gf, WM and age, and testing them allows us to see in which configuration the model is better fitted to the empirical data. Consequently, this permits an evaluation of: (1) whether age is an independent predictor of Gf, or whether it operates through WM, and (2) whether WM is a true predictor of Gf, or whether their observed association is due to the association of both these variables with age. When we compare these two tested models, some parameters will be the same. For example, the variance of Gf explained by a model including an independent variable and a mediator will be the same whether age is the independent variable and WM the mediator, or conversely. However, other parameters will vary, indicating different fits of the models to the data, including an increase in the explained variance of Gf after adding a mediator to a model with only a predictor. This means that by changing the position of individual variables we obtain models reflecting different relationships between variables. Consequently, it is possible to assess which one has a better fit to the empirical data. In light of the presented results, the model in which age acts as a mediator (source of apparent correlation) has a certain advantage. 
Interpreting together the two mediation models conducted, it can be concluded that (1) age is a significant independent predictor of Gf, with WM not mediating this relationship, while (2) age explains the observed relationship between WM and Gf, which disappears when the variability explained by age is removed.

Regarding the mediating role of WM in the relationship between age and Gf, as indicated in the introduction, many studies confirm (a) the relationship between WM and Gf, or WM's contribution to explaining Gf variability (e.g., Ackerman et al., 2005; Oberauer et al., 2005; Kane et al., 2005) and (b) the decline with age of both Gf and WM (e.g., Harada et al., 2013; Hartshorne \& Germine, 2015; Salthouse 2016). However, the often-demonstrated contribution of WM to explaining Gf is not the same as WM playing a mediating role in the changes in Gf that occur with age. As mentioned in the introduction, the mediating role of WM in the relationship between age and Gf is not a well-documented phenomenon. In the 1990s, Salthouse studied quite extensively the mediating role of various aspects of cognitive functioning, including WM, between age and fluid ability. In an article on this issue, he reported three studies, only one of which found WM to explain a significant range of variance in fluid ability after accounting for processing speed (Salthouse, 1991). Consequently, Salthouse (1996b) found processing speed to be the factor explaining the deterioration of fluency abilities with age. Results of later studies on the mediators of the relationship between age and Gf provided mixed results. Study using SEM showed an unambiguous mediating role of WM between age and Gf, while contradicting the mediating role of processing speed (Chen \& Li, 2007). Other study among age-diverse individuals (from childhood to old age), also using SEM, found that the mediating role of WM may change with age and WM is an independent mediator between age and Gf only in individuals over 55 years old (Nettelbeck \& Burns, 2010). In contrast, in yet another study that included participants ranging in age from adolescence to old age and that used hierarchical regression analysis, WM ceased to contribute to explaining Gf when age and processing speed were simultaneously included in the model, with the latter proving most significant (Schretlen et al., 2000).

Salthouse, based on a number of studies conducted, argued for the existence of a general aging factor. He pointed out that the changes observed in particular aspects of cognitive functioning with age are not independent of each other, and that on average half of the effect of age on a given measure of cognitive function is shared with other measures of cognitive function, even if they involve quite different aspects of cognitive functioning (Salthouse, 1996a; Salthouse et al., 1998). The existence of a general cognitive factor of aging is also clearly indicated by the results of a relatively recent and manuscript-referenced meta-analysis involving a total of measurements from 30,000 individuals (Tucker-Drob et al., 2019). Findings revealing the presence 
of a general aging factor suggest that the influence of age is the source of variance shared by different cognitive functions measured in individuals at different stages of development. Of course, this factor is not age per se, and there may be many underlying mechanisms that have not yet been identified. Consistent with reports of the existence of a general factor of cognitive ageing are the results of the second mediation analysis reported in this article, indicating that age explains the positive relationship between WM and Gf.

At the same time, it should be noted that the results of the mediation analyses presented here could be referred to two different perspectives in studying the relationship between WM, Gf, and age. From a developmental psychology perspective, it can be assumed that working memory is not a mediator between age and fluid intelligence (the first mediation model), whereas there are grounds to infer the existence of a general factor of cognitive aging (second mediation model). In turn, from a methodological perspective, the obtained results (the second mediation model) demonstrate that when the relationship between WM and Gf in individuals of varying ages is examined, the age variable may be a source of apparent correlation. Therefore, in such cases, it would be appropriate to control for the age contribution to the relationship being analysed.

\section{LIMITATIONS}

The presented study is not free from limitations. In evaluating developmental differences between early and late adulthood, the cross-sectional comparisons were used, when longitudinal studies provide better information on developmental changes. In addition, the study included individuals in early and late adulthood, but lacked participants in middle adulthood. The participation of persons from only two age groups was due to the objectives of the broader project from which the data analysed were derived. Finally, only one type of task measuring WM was used. Using a broader spectrum of WM measures designed to assess different aspects of WM would have helped to deepen the implications of the findings.

\section{CONCLUSIONS}

The analyses presented in the article focused on the role of age in the relationship between WM and Gf. Although WM and Gf were found to be weaker among older 
than among young adults, age was not a significant moderator of the association between WM and Gf. The relationship between age and Gf was found to not be mediated by WM. At the same time, age fully mediated the relationship between WM and Gf, implying that there is a general factor of cognitive aging and that it may be the source of the presence of an apparent relationship between WM and Gf in age-diverse groups.

\section{REFERENCES}

Ackerman, P. L., Beier, M. E., \& Boyle, M. O. (2005). Working memory and intelligence: The same or different constructs? Psychological Bulletin, 131(1), 30-60. https://doi.org/10.1037/00332909.131.1.30

Aiken, L. S., \& West, S. G. (1991). Multiple regression: Testing and interpreting interactions. SAGE Publications. https://doi.org/10.1016/0886-1633(93)90008-d

Akoglu, H. (2018). User's guide to correlation coefficients. Turkish Journal of Emergency Medicine, 18(3), 91-93. https://doi.org/10.1016/j.tjem.2018.08.001

Barbey, A. K., Colom, R., Paul, E. J., \& Grafman, J. (2014). Architecture of fluid intelligence and working memory revealed by lesion mapping. Brain Structure and Function, 219(2), 485-494. https://doi.org/10.1007/s00429-013-0512-z

Basak, C., \& Verhaeghen, P. (2011). Aging and switching the focus of attention in working memory: Age differences in item availability but not in item accessibility. Journals of Gerontology - Series B Psychological Sciences and Social Sciences, 66 B(5), 519-526. https://doi.org/10.1093/geronb/ gbr028

Bopp, K. L., \& Verhaeghen, P. (2005). Aging and verbal memory span: A meta-analysis. Journals of Gerontology - Series B Psychological Sciences and Social Sciences, 60(5), 223-233. https://doi. org/10.1093/geronb/60.5.P223

Borella, E., Carretti, B., \& De Beni, R. (2008). Working memory and inhibition across the adult lifespan. Acta Psychologica, 128(1), 33-44. https://doi.org/10.1016/j.actpsy.2007.09.008

Braver, T. S., \& West, R. (2008). Working memory, executive control, and aging. In F. I. Craik \& T. A. Salthouse (Eds.), The handbook of aging and cognition (3rd ed.) (pp. 311-372). Psychology Press.

Burgess, G. C., Gray, J. R., Conway, A. R. A., \& Braver, T. S. (2011). Neural mechanisms of interference control underlie the relationship between fluid intelligence and working memory span. Journal of Experimental Psychology: General, 140(4), 674-692. https://doi.org/10.1037/a0024695

Burgoyne, A. P., Hambrick, D. Z., \& Altmann, E. M. (2019). Is working memory capacity a causal factor in fluid intelligence? Psychonomic Bulletin and Review, 26(4), 1333-1339. https://doi. org/10.3758/s13423-019-01606-9

Cabeza, R., \& Dennis, N. A. (2012). Frontal lobes and aging. Deterioration and compensation. In D. T. Stuss \& R. T. Knight (Eds.), Principles of Frontal Lobe Function (2nd ed., pp. 628-652). Oxford University Press. https://doi.org/10.1093/acprof:oso/9780195134971.001.0001

Chatham, C. H., Herd, S. A., Brant, A. M., Hazy, T. E., Miyake, A., O’Reilly, R., \& Friedman, N. P. (2011). From an executive network to executive control: A computational model of the n-back task. Journal of Cognitive Neuroscience, 23(11), 3598-3619. https://doi.org/10.1162/jocn_a_00047 
Chen, T., \& Li, D. (2007). The roles of working memory updating and processing speed in mediating age-related differences in fluid intelligence. Aging, Neuropsychology, and Cognition, 14(6), 631-646. https://doi.org/10.1080/13825580600987660

Chen, Y. N., Mitra, S., \& Schlaghecken, F. (2008). Sub-processes of working memory in the N-back task: An investigation using ERPs. Clinical Neurophysiology, 119(7), 1546-1559. https://doi. org/10.1016/j.clinph.2008.03.003

Chuderski, A. (2014). How well can storage capacity, executive control, and fluid reasoning explain insight problem solving. Intelligence, 46(1), 258-270. https://doi.org/10.1016/j.intell.2014.07.010

Chuderski, A. (2015). The broad factor of working memory is virtually isomorphic to fluid intelligence tested under time pressure. Personality and Individual Differences, 85, 98-104. https://doi. org/10.1016/j.paid.2015.04.046

Chuderski, A., \& Nęcka, E. (2012). The contribution of working memory to fluid reasoning: Capacity, control, or both? Journal of Experimental Psychology: Learning Memory and Cognition, 38(6), 1689-1710. https://doi.org/10.1037/a0028465

Chuderski, A., Taraday, M., Nęcka, E., \& Smoleń, T. (2012). Storage capacity explains fluid intelligence but executive control does not. Intelligence, 40(3), 278-295. https://doi.org/10.1016/j. intell.2012.02.010

Cochrane, A., Simmering, V., \& Green, S. (2019). Fluid intelligence is related to capacity in memory as well as attention: Evidence from middle childhood and adulthood. PloS ONE, 14(8), Article e0221353. https://doi.org/10.1371/journal.pone.0221353

Colom, R., Abad, F. J., Quiroga, M. Á., Shih, P. C., \& Flores-Mendoza, C. (2008). Working memory and intelligence are highly related constructs, but why? Intelligence, 36(6), 584-606. https://doi. org/10.1016/j.intell.2008.01.002

Colom, R., Abad, F. J., Rebollo, I., \& Shih, P. C. (2005). Memory span and general intelligence: A latent-variable approach. Intelligence, 33(6), 623-642. https://doi.org/10.1016/j. intell.2005.05.006

Colom, R., Flores-Mendoza, C., \& Rebollo, I. (2003). Working memory and intelligence. Personality and Individual Differences, 34(1), 33-39. https://doi.org/10.1017/9781108770422.022

Colom, R., Privado, J., García, L. F., Estrada, E., Cuevas, L., \& Shih, P. C. (2015). Fluid intelligence and working memory capacity: Is the time for working on intelligence problems relevant for explaining their large relationship? Personality and Individual Differences, 79, 75-80. https://doi.org/10.1016/j.paid.2015.01.051

Colom, R., Rubio, V. J., Shih, P. C., \& Santacreu, J. (2006). Fluid intelligence, working memory and executive functioning. Psicothema, 18(4), 816-821.

Conway, A. R. A, Cowan, N., Bunting, M. F., Therriault, D. J., \& Minkoff, S. R. B. (2002). A latent variable analysis of working memory capacity, short-term memory capacity, processing speed, and general f luid intelligence. Intelligence, 30(2), 163-184. https://doi.org/10.1016/S01602896(01)00096-4

Cowan, N., Fristoe, N. M., Elliott, E. M., Brunner, R. P., \& Saults, J. S. (2006). Scope of attention, control of attention, and intelligence in children and adults. Memory and Cognition, 34(8), 1754-1768. https://doi.org/10.3758/BF03195936

Dang, C. P., Braeken, J., Colom, R., Ferrer, E., \& Liu, C. (2014). Why is working memory related to intelligence? Different contributions from storage and processing. Memory, 22(4), 426-441. https://doi.org/10.1080/09658211.2013.797471

Dehn, M. J. (2017). How working memory enables fluid reasoning. Applied Neuropsychology: Child, 6(3), 245-247. https://doi.org/10.1080/21622965.2017.1317490 
Dodson, C. S., Bawa, S., \& Krueger, L. E. (2007). Aging, metamemory, and high-confidence errors: A misrecollection account. Psychology and Aging, 22(1), 122-133. https://doi.org/10.1037/08827974.22.1.122

Engle, R. W., \& Kane, M. J. (2004). Executive attention, working memory capacity, and a two-factor theory of cognitive control. The Psychology of Learning and Motivation, 44, 145-199. https://doi.org/10.1016/S0079-7421(03)44005-X

Engle, R. W., Kane, M. J., \& Tuholski, S. W. (1999a). Individual differences in working memory capacity and what they tell us about controlled attention, general fluid intelligence, and functions of the prefrontal cortex. In A. Miyake \& P. Shah (Eds.), Models of working memory: Mechanisms of active maintenance and executive control (pp. 102-134). Cambridge University Press. https://doi.org/10.1037/a0021324

Engle, R. W., Tuholski, S. W., Laughlin, J. E., \& Conway, A. R. A. (1999b). Working memory, shortterm memory, and general fluid intelligence: A latent-variable approach. Journal of Experimental Psychology. General, 128(3), 309-331. https://doi.org/10.1037/0096-3445.128.3.309

Eriksson, J., Vogel, E. K., Lansner, A., Bergström, F., \& Nyberg, L. (2015). Neurocognitive architecture of working memory. Neuron, 88(1), 33-46. https://doi.org/10.1016/j.neuron.2015.09.020

Faul, F., Erdfelder, E., Lang, A. G., \& Buchner, A. (2007). G*Power 3: A flexible statistical power analysis program for the social, behavioral, and biomedical sciences. Behavior Research Methods, 39(2), 175-191. https://doi.org/10.3758/BF03193146

Filippi, R., Ceccolini, A., Periche-Tomas, E., \& Bright, P. (2020). Developmental trajectories of metacognitive processing and executive function from childhood to older age. Quarterly Journal of Experimental Psychology, 73(11), 1757-1773. https://doi.org/10.1177/1747021820931096

Frischkorn, G. T., \& Oberauer, K. (2021). Intelligence test items varying in capacity demands cannot be used to test the causality of working memory capacity for fluid intelligence. Psychonomic Bulletin and Review, 28, 1423-1432. https://doi.org/10.3758/s13423-021-01909-w

Fry, A. F., \& Hale, S. (1996). Processing speed, working memory, and fluid intelligence: Evidence for a Developmental Cascade. Psychological Science, 7, 237-241. https://doi. org/10.1111/j.1467-9280.1996.tb00366.x

Fukuda, K., Vogel, E., Mayr, U., \& Awh, E. (2010). Quantity, not quality: The relationship between fluid intelligence and working memory capacity. Psychonomic Bulletin \& Review, 17(5), 673-679. https://doi.org/10.3758/17.5.673

Gray, J. R., Chabris, C. F., \& Braver, T. S. (2003). Neural mechanisms of general fluid intelligence. Nature Neuroscience, 6(3), 316-322. https://doi.org/10.1038/nn1014

Harada, C. N., Love, M. C. N., \& Triebel, K. (2013). Normal cognitive aging. Clinics in Geriatric Medicine, 29(4), 737-752. https://doi.org/10.1016/j.cger.2013.07.002

Harrison, T. L., Shipstead, Z., \& Engle, R. W. (2015). Why is working memory capacity related to matrix reasoning tasks? Memory \& Cognition, 43(3), 389-396. https://doi.org/10.3758/s13421014-0473-3

Hartshorne, J. K., \& Germine, L. T. (2015). When does cognitive functioning peak? The asynchronous rise and fall of different cognitive abilities across the lifespan. Psychological Science, 26(4), 433-443. https://doi.org/10.1177/0956797614567339

Hasher, L., \& Zacks, R. T. (1988). Working memory, comprehension, and aging: A review and a new view. In G. H. Bower (Ed.), The psychology of learning and motivation (pp. 193-225). Academic Press. https://doi.org/https://doi.org/10.1016/S0079-7421(08)60041-9

Hayes, A. F. (2018). Introduction to mediation, moderation, and conditional process analysis. A regression-based approach. The Guilford Press. 
Hertzog, C., \& Dunlosky, J. (2011). Metacognition in later adulthood: Spared monitoring can benefit older adults' self-regulation. Current Directions in Psychological Science, 20(3), 167-173. https://doi.org/10.1177/0963721411409026

Jaeggi, S. M., Buschkuehl, M., Perrig, W. J., \& Meier, B. (2010). The concurrent validity of the $N$-back task as a working memory measure. Memory, 18(4), 394-412. https://doi. org/10.1080/09658211003702171

Jaroslawska, A. J., \& Rhodes, S. (2019). Adult age differences in the effects of processing on storage in working memory: A meta-analysis. Psychology and Aging, 34(4), 512-530. https://doi. org/10.1037/pag0000358

Jastrzębski, J., Ciechanowska, I., \& Chuderski, A. (2018). The strong link between fluid intelligence and working memory cannot be explained away by strategy use. Intelligence, 66, 44-53. https:// doi.org/10.1016/j.intell.2017.11.002

Kane, M. J., Conway, A. R. A., Miura, T. K., \& Colflesh, G. J. (2007). Working memory, attention control, and the N-back task: A question of construct validity. Journal of Experimental Psychology: Learning, Memory, and Cognition, 33(3), 615-622. https://pubmed.ncbi.nlm.nih.gov/17470009

Kane, M. J., \& Engle, R. W. (2002). The role of prefrontal cortex in working-memory capacity, executive attention, and general fluid intelligence: An individual-differences perspective. Psychonomic Bulletin \& Review, 9(4), 637-671. https://doi.org/10.3758/BF03196323

Kane, M. J., Hambrick, D. Z., \& Conway, A. (2005). Working memory capacity and fluid intelligence are strongly related constructs: Comment on Ackerman, Beier, and Boyle (2005). Psychological Bulletin, 131(1), 66-71. https://doi.org/10.1037/0033-2909.131.1.66

Kovacs, K., \& Conway, A. R. A. (2016). Process overlap theory: A unified account of the general factor of intelligence. Psychological Inquiry, 27(3), 151-177. https://doi.org/10.1080/104784 0X.2016.1153946

Kyllonen, P. C., \& Christal, R. E. (1990). Reasoning ability is (little more than) working-memory capacity?! Intelligence, 14(4), 389-433. https://doi.org/10.1016/S0160-2896(05)80012-1

Lakens, D. (2013). Calculating and reporting effect sizes to facilitate cumulative science: A practical primer for t-tests and ANOVAs. Frontiers in Psychology, 4, 863. https://doi.org/10.3389/ fpsyg. 2013.00863

Lustig, C., Hasher, L., \& Zacks, R. T. (2007). Inhibitory deficity theory: Recent developments in a "new view". In D. S. Gorfein \& C. M. MacLeod (Eds.), Inhibition in cognition (pp. 145-162). American Psychological Association.

Manard, M., Carabin, D., Jaspar, M., \& Collette, F. (2014). Age-related decline in cognitive control: the role of fluid intelligence and processing speed. BMC Neuroscience, 15, Article 7. https://doi. org/10.1186/1471-2202-15-7

Nęcka, E., \& Lulewicz, A. (2016). Capacity, control, or both - Which aspects of working memory contribute to children's general fluid intelligence? Polish Psychological Bulletin, 47(1), 21-28. https://doi.org/10.1515/ppb-2016-0003

Nettelbeck, T., \& Burns, N. R. (2010). Processing speed, working memory and reasoning ability from childhood to old age. Personality and Individual Differences, 48(4), 379-384. https://doi. org/10.1016/j.paid.2009.10.032

Oberauer, K., Wilhelm, O., Schulze, R., \& Süß, H. M. (2005). Working memory and intelligence - Their correlation and their relation: Comment on Ackerman, Beier, and Boyle (2005). Psychological Bulletin, 131(1), 61-65. https://doi.org/10.1037/0033-2909.131.1.61

Peirce, J. W. (2009). Generating stimuli for neuroscience using PsychoPy. Frontiers in Neuroinformatics, 2, 10. https://doi.org/10.3389/neuro.11.010.2008 
Rey-Mermet, A., Gade, M., Souza, A. S., von Bastian, C. C., \& Oberauer, K. (2019). Is executive control related to working memory capacity and fluid intelligence? Journal of Experimental Psychology: General, 148(8), 1335-1372. https://doi.org/10.1037/xge0000593

Rose, N. S. (2013). Individual differences in working memory, secondary memory, and fluid intelligence: Evidence from the levels-of-processing span task. Canadian Journal of Experimental Psychology, 67(4), 260-270. https://doi.org/10.1037/a0034351

Salthouse, T. A. (1991). Mediation of adult age differences in cognition by reductions in working memory and speed of processing. Psychological Science, 2(3), 179-183. https://doi. org/10.1111/j.1467-9280.1991.tb00127.x

Salthouse, T. A. (1996a). Constraints on theories of cognitive aging. Psychonomic Bulletin \& Review, 3(3), 287-299. https://doi.org/10.3758/BF03210753

Salthouse, T. A. (1996b). The processing-speed theory of adult age differences in cognition. Psychological Review, 103(3), 403-428. https://doi.org/10.1037/0033-295X.103.3.403

Salthouse, T. A. (2009). When does age-related cognitive decline begin? Neurobiology of Aging, 30(4), 507-514. https://doi.org/10.1016/j.neurobiolaging.2008.09.023

Salthouse, T. A. (2011). Consequences of age-related cognitive declines. Annual Review of Psychology, 63, 201-226. https://doi.org/10.1146/annurev-psych-120710-100328

Salthouse, T. A. (2014). Relations between running memory and fluid intelligence. Intelligence, 43, 1-7. https://doi.org/10.1016/j.intell.2013.12.002

Salthouse, T. A. (2016). Continuity of cognitive change across adulthood. Psychonomic Bulletin \& Review, 23(3), 932-939. https://doi.org/10.3758/s13423-015-0910-8

Salthouse, T. A., Fristoe, N., McGuthry, K. E., \& Hambrick, D. Z. (1998). Relation of task switching to speed, age, and fluid intelligence. Psychology and Aging, 13(3), 445-461. https://doi. org/10.1037/0882-7974.13.3.445

Salthouse, T. A., \& Pink, J. E. (2008). Why is working memory related to fluid intelligence? Psychonomic Bulletin \& Review, 15(2), 364-371.

Schmiedek, F., Lövdén, M., \& Lindenberger, U. (2014). A task is a task is a task: Putting complex span, $n$-back, and other working memory indicators in psychometric context. Frontiers in Psychology, 5, Article 1475. https://doi.org/10.3389/fpsyg.2014.01475

Schretlen, D., Pearlson, G. D., Anthony, J. C., Aylward, E. H., Augustine, A. M., Davis, A., \& Barta, P. (2000). Elucidating the contributions of processing speed, executive ability, and frontal lobe volume to normal age-related differences in fluid intelligence. Journal of the International Neuropsychological Society, 6(1), 52-61. https://doi.org/10.1017/S1355617700611062

Sheehan, D. V., Lecrubier, Y., Sheehan, K. H., Amorim, P., Janavs, J., Weiller, E., Hergueta, T., Baker, R., \& Dunbar, G. C. (1998). The Mini-International Neuropsychiatric Interview (M.I.N.I.): The development and validation of a structured diagnostic psychiatric interview for DSM-IV and ICD-10. The Journal of clinical psychiatry, 59 Suppl 20, 22-57.

Shelton, J. T., Elliott, E. M., Matthews, R. A., Hill, B. D., \& Gouvier, W. D. (2010). The relationships of working memory, secondary memory, and general fluid intelligence: Working memory is special. Journal of Experimental Psychology. Learning, Memory, and Cognition, 36(3), 813-820. https://doi.org/10.1037/a0019046

Shipstead, Z., Harrison, T. L., \& Engle, R. W. (2016). Working memory capacity and fluid intelligence: Maintenance and disengagement. Perspectives on Psychological Science, 11(6), 771-799. https://doi.org/10.1177/1745691616650647

Shipstead, Z., Redick, T. S., Hicks, K. L., \& Engle, R. W. (2012). The scope and control of attention as separate aspects of working memory. Memory, 20(6), 608-628. https://doi.org/10.1080/0965 8211.2012 .691519 
Smolen, T., \& Chuderski, A. (2015). The quadratic relationship between difficulty of intelligence test items and their correlations with working memory. Frontiers in Psychology, 24(6), Article 1270. https://doi.org/10.3389/fpsyg.2015.01270

Waris, O., Soveri, A., Ahti, M., Hoffing, R. C., Ventus, D., Jaeggi, S. M., Seitz, A. R., \& Laine, M. (2017). A latent factor analysis of working memory measures using large-scale data. Frontiers in psychology, 8, Article 1062. https://doi.org/10.3389/fpsyg.2017.01062

Stanislaw, H., \& Todorov, N. (1999). Calculation of signal detection theory measures. Behavior Research Methods, Instruments, \& Computers, 3(1), 137-149.

Süß, H. M., Oberauer, K., Wittmann, W. W., Wilhelm, O., \& Schulze, R. (2002). Working-memory capacity explains reasoning ability - And a little bit more. Intelligence, 30(3), 261-288. https://doi.org/10.1016/S0160-2896(01)00100-3

Thomas, P., Rammsayer, T., Schweizer, K., \& Troche, S. (2015). Elucidating the functional relationship between working memory capacity and psychometric intelligence: A fixed-links modeling approach for experimental repeated-measures designs. Advances in Cognitive Psychology, 11(1), 3-13. https://doi.org/10.5709/acp-0166-6

Tucker-Drob, E. M., Brandmaier, A. M., \& Lindenberger, U. (2019). Coupled cognitive changes in adulthood: A meta-analysis how many causes are there of aging-related decrements in cognitive functioning? Psychological Bulletin, 145(3), 273-301. https://doi.org/10.1037/bul0000179

Unsworth, N., Fukuda, K., Awh, E., \& Vogel, E. K. (2014). Working memory and fluid intelligence: Capacity, attention control, and secondary memory retrieval. Cognitive Psychology, 71, 1-26. https://doi.org/10.1016/j.cogpsych.2014.01.003

Unsworth, N., \& Spillers, G. J. (2010). Working memory capacity: Attention control, secondary memory, or both? A direct test of the dual-component model. Journal of Memory and Language, 62(4), 392-406. https://doi.org/10.1016/j.jml.2010.02.001

Unsworth, N., Spillers, G. J., \& Brewer, G. A. (2009). Examining the relations among working memory capacity, attention control, and fluid intelligence from a dual-component framework. Psychology Science, 51(4), 388-402.

West, R. (1996). An application of prefrontal cortex function theory to cognitive aging. Psychological Bulletin, 120(2), 272-292. https://doi.org/10.1037/0033-2909.120.2.272

Wiley, J., Jarosz, A. F., Cushen, P. J., \& Colflesh, G. J. H. (2011). New rule use drives the relation between working memory capacity and Raven's Advanced Progressive Matrices. Journal of Experimental Psychology: Learning Memory and Cognition, 37(1), 256-263. https://doi.org/10.1037/ a0021613

Zakrzewski, A. C., Sanders, E. C., \& Berry, J. M. (2021). Evidence for Age-Equivalent and Task-Dissociative Metacognition in the Memory Domain. Frontiers in psychology, 12, Article 630143. https://doi.org/10.3389/fpsyg.2021.630143 